\title{
NEUE REGELUNGEN DES INTERNATIONALEN ZIVILVERFAHRENSRECHTS IN BRASILIEN
}

RECENT DEVELOPMENTS IN BRAZILIAN INTERNATIONAL CIVIL PROCEDURE

Emília Lana de Freitas Castro

Dozentin für Internationales Privatrecht an der Universität des Bundeslandes Rio de Janeiro. Master-Anwärterin des Fachs Internationales Recht an der Universität des Bundeslandes Rio de Janeiro. Rechtsanwältin.

Ely Caetano Xavier Junior

Dozent für Internationales Privatrecht an der Universität des Bundeslandes Rio de Janeiro. Master-Anwärter des Fachs Internationales Recht an der Universität des Bundeslandes Rio de Janeiro und der Universität London. Rechtsanwalt.

Zusammenfassung: Aufgrund des voranschreitenden Reformprozesses im Hinblick auf die brasilianische Zivilprozessordnung finden gegenwärtig zahlreiche Veränderungen Einzug in das internationale Zivilverfahrensrecht. Unter anderem sieht ein Gesetzentwurf des brasilianischen Nationalkongresses Änderungen bei der internationalen Zuständigkeitsregelungen und der Wahl des Gerichtsstandes vor. Dies wird in Zukunft relevant bei internationalen Wirtschaftstransfers sein. Ziel dieser Arbeit ist es, durch eine genauere Analyse der Lehre und Rechtsprechung, die Unterschiede zwischen der aktuellen Zivilprozessordnung und dem Gesetzesvorhaben herauszuarbeiten.

Schlüsselworte: Brasilianische Zivilprozessordnung - internationale Zuständigkeit - Wahl des internationalen Gerichtsstandes.

Abstract: The international civil procedure is undergoing transformations in Brazil, due to the project of the new Civil Procedure Code, which is still pending enactment by the National Congress. Among several alterations, the project will bring changes regarding the rules of international jurisdiction and choice of forum, which are of utmost importance for international business transactions. Therefore, scholar and jurisprudential controversies about these institutes are analyzed, considering both the current Civil Procedure Code and the project still under consideration by the Congress. 
Keywords: Brazilian Civil Procedure Code - international jurisdiction - choice of forum.

\section{EINFÜHRUNG}

JuristischeBeziehungen, dieinternationaleElemente einbeziehen, führen zu zwei Fragestellungen: die Frage nach der Zuständigkeit und die Frage nach dem anzuwendenden Recht. Die Bestimmung des zuständigen Gerichts stellt dabei die erste Herausforderung für die Parteien dar. Im Idealfall legt die nationale Rechtsordnung fest, welche Gerichte für bestimmte Streitsachen zuständig sind. Dies erwirkt ein gewisses Maß an Rechtssicherheit bei den Rechtssubjekten. Die internationale Zuständigkeit zeigt sich daher als grundlegendes Institut des internationalen Zivilverfahrensrechts.

Parallel zur internationalen Zuständigkeit erlauben die Rechtsordnungen des Öfteren, dass die Parteien - aufgrund ihrer Willensautonomie - die Zuständigkeit der Gerichte teilweise abbedingen oder auswählen können. Die Wahl des Gerichtsstandes ist entscheidend, weil sie die gesetzliche vorgeschriebene Zuständigkeit eines Gerichtes auf internationalen Ebenen verändern kann.

In diesem Sinne versuchen wir, die zukünftigen Änderungen dieser grundlegenden Institute des internationalen Zivilverfahrensrechts, welche sich momentan im Entstehen befinden, zu beleuchten ${ }^{1}$. Zu diesem Zweck ziehen wir eine Vergleichsparallele zwischen den Regelungen der internationalen Zuständigkeit und der Wahl des Gerichtsstandes der heutigen Zivilprozessordnung und denen, welche der zukünftigen Rechtslage entsprechen. Wir hoffen, dass dieser Vergleich dazu beitragen wird, die Entwicklung des internationalen Zivilprozesses in Brasilien im Rahmen der Rechtslehre und der Rechtsprechung zu veranschaulichen.

\section{INTERNATIONALE ZUSTÄNDIGKEIT}

Die Artikel 88 und 89 der brasilianischen Zivilprozessordnung und ihre jeweiligen Absätze legen Voraussetzungen fest, unter denen die brasilianischen Gerichte ihre Gerichtsbarkeit ausüben können oder müssen. Obwohl die Redaktion der Vorschrift sich wortwörtlich mit „,internationaler Zuständigkeit“" bei der Zuständigkeitseinschränkung der nationalen oder internationalen Gerichte ausdrückt, wollen wir daran erinnern, dass dieser Ausdruck in sich selbst missverständlich ist.

In der Regel übt der Staat seine Gerichtsbarkeit bei

1 In unseren Kommentaren benutzen wir als Referenz die Version des Projektes der Zivilprozessordnung (Gesetzesprojekt Nr. 6025/2005) vom November 2012, bereits genehmigt durch die Sonderkommission des Nationalkongress. 
Interessenkonflikten aus. Die Gerichtsbarkeit veräußert daher diese staatliche Macht ${ }^{2}$. Die Funktion der Gerichtsbarkeit wird abstrakt und uneingeschränkt an alle Organe der Judikative zugeteilt. Dabei fließt sie allmählich durch einen Konkretisierungsprozess bis sie an das für die Entscheidung des Konfliktes kompetente Gerichtsorgan gelangt. Auf dieser Weise und durch Zuständigkeitsregeln wird die Gerichtsbarkeit innerhalb ihrer ausübenden Organen verteilt ${ }^{3}$.

In diesem Sinne bezieht sich die in der Zivilprozessordnung erwähnte internationale Zuständigkeit nicht auf ein technisches Zuständigkeitskonzept, sondern auf die jeweilige Definition von Gerichtsbarkeit ${ }^{4}$. Der Grund dieser Bestätigung ist, dass die Artikel 88 und 89 die Fälle aufführen, in denen die brasilianische Judikative zuständig ist ${ }^{5}$. Dabei soll hervorgehoben werden, dass die hiermit analysierten Vorschriften sich nicht auf eine spezifische Übertragung eines Teils der Gerichtsbarkeit auf ein bestimmtes Gerichtsorgan beziehen. Erst nach Überprüfung der Ausübungsmöglichkeiten der brasilianischen Gerichtsbarkeit wird die Rechtsordnung detaillierter die Umstände aufzeigen und festlegen, wann ein bestimmtes Gerichtsorgan zuständig wird. Somit wird die Zuständigkeit bestimmt ${ }^{6}$.

Wichtig ist es, hervorzuheben, dass die bestimmenden Normen der Prozessordnungen - als Normen des Öffentlichen Rechts - auch unilaterale Normen sind, das heißt, die Artikel 88 und 89 der Zivilprozessordnung können nicht zur Festlegung der Gerichtsbarkeit einer ausländischen Gerichtsgewalt dienen. Im Gegenteil: der brasilianische Gesetzgeber würde damit in die Rechtshoheit des ausländischen Staates eindringen. Gemäß Celso Agricola Barbi:

\section{Die Gerichtsbarkeit erleidet eine Einschränkung ab dem Moment in der sie effektiv in einer anderen Gerichtsbarkeit einwirkt: es handelt sich dabei um das Effektivitätsprinzip, das heißt, die Macht, das Urteil zu vollstrecken, zumal es andere Länder gibt, die ebenfalls das Urteil in ihrem Lande nicht anerkennen und daraufhin dessen Vollstreckung in}

2 CINTRA, Antonio Carlos de Araújo; GRINOVER, Ada Pellegrini; DINAMARCO, Cândido Rangel. Teoria Geral do Processo. 23. Auflage. São Paulo: Malheiros, 2007, Seite 30.

3 CINTRA, Antonio Carlos de Araújo; GRINOVER, Ada Pellegrini; DINAMARCO, Cândido Rangel. Teoria Geral do Processo. 23. Auflage. São Paulo: Malheiros, 2007, Seite 248.

4 Aufgrund der Ratifizierung des Gebrauchs des Ausdruckes Kompetenz durch die Rechtslehre und Rechtsprechung erlauben wir uns dessen Anwendung während des gesamten Textes.

5 CINTRA, Antonio Carlos de Araújo; GRINOVER, Ada Pellegrini; DINAMARCO, Cândido Rangel. Teoria Geral do Processo. 23. Auflage. São Paulo: Malheiros, 2007, Seite 249.

6 TIBURCIO, Carmen. Temas de Direito Internacional. Rio de Janeiro: Renovar, 2006, Seite 85. 
ihrem Lande nicht durchführen würden. Dies würde zur faktischen Unwirksamkeit des Urteils führen?

Somit ist es unangebracht, die Ausübung der Gerichtsbarkeit durch einen ausländischen Staat aufgrund des in den Artikeln 88 und 89 der Zivilprozessordnung falschen Bilateralismus festzulegen. Die Behauptung jedoch, dass ein brasilianischer Richter auf der Grundlage fehlender Effektivität seine Gerichtsbarkeit in einer bestimmten Streitsache nicht ausüben dürfte, ist möglich. In diesem Falle wäre sein Urteil im Ausland nicht vollstreckbar.

\subsection{Ausdrückliche Annahmen der Zivilprozessordnung zur Ausübung der brasilianischen Gerichtsbarkeit}

Der Artikel 88 der Zivilprozessordnung nennt die Alternativen bei denen eine konkurrierende Zuständigkeit besteht. Das heißt, der Artikel 88 erlaubt, dass verschiedene Rechtssachen auch durch ausländische Gerichte entschieden werden. Demzufolge kann das im Ausland ausgesprochene Urteil - unter der Voraussetzung, dass es mindestens eine der drei Fällen ${ }^{8}$ des Artikels 88 entspricht - in Brasilien gültig und vollstreckbar sein. Voraussetzung ist, dass das Urteil von dem Obersten Gerichtshof (Superior Tribunal de Justiça - STJ), gemäß den Anforderungen der Artikel 15 und 17 des Einführungsgesetzes der brasilianischen Gesetzesnormen (Lei de Introdução às Normas de Direito Brasileiro - LINDB) anerkannt wird ${ }^{9}$. Diese Anforderungen beziehen sich auf die Notwendigkeit, dass das Urteil durch einen

7 BARBI, Celso Agricola. Comentários do Código de Processo Civil.9. Auflage. Rio de Janeiro: Forense, 1994, Seite 239.

8 Laut José Carlos Barbosa Moreira, sind die Voraussetzungen der internationalen Zuständigkeit nicht kumulativ sondern alternativ: "eine jede genüge für sich alleine. Die brasilianische Justiz ist daher zuständig, wenn der Beklagte in Brasilien wohnhaft ist, selbst wenn die Rechtssache infolge einer im Ausland geschehenen Tat verursacht wurde. Das Gleiche gilt für den Fall, wenn die Strafauflage in Brasilien zu vollziehen ist und der Angeklagte im Ausland wohnhaft ist.” In MOREIRA, José Carlos Barbosa. Probleme in Bezug auf internationale Streitigkeiten. In Temas de Direito Processual. 5. Serie. Rio de Janeiro: Saraiva. 1994, Seite 140. Der Oberste Gerichtshof (STJ) äußerte sich bereits in diesem Sinne: "Internationale Zuständigkeit - zusammenhängende Rechtssachen. Die Zuständigkeit des brasilianischen Gerichts liegt vor wenn eine der in den Artikeln 88 und 89 der Zivilprozessordnung vorgesehenen Annahmen vorliegt. Das brasilianische Recht wählte nicht den Zusammenhang als Kriterium zur Festlegung der internationalen Zuständigkeit die, aufgrund dessen, auch nicht fortführbar ist". (Oberster Gerichtshof, Revision Nr. 2170/SP, Berichterstatter: Minister Eduardo Ribeiro. Tagesanzeiger der Justiz vom 30.09.1990, Seite 08842, Zeitschrift des Obersten Gerichtshofes, siehe 00012, Seite 00361).

9 Gesetzesverordnung Nr. 4.657 vom 4. September 1942 mit der Redaktion des Gesetzes 12.376 vom 2010 . 
zuständigen Richter ausgesprochen wurde, gemäß der Gesetzgebung des Ursprungslandes. Zur Anerkennung ist es ebenso wichtig, dass die beteiligten Parteien geladen wurden oder ein Versäumnisurteil ergangen ist; dass das Urteil rechtskräftig wurde; dass das Urteil im verurteilenden Land vollstreckbar ist; und, schließlich, dass das ausländische Urteil nicht die brasilianische Rechtshoheit, die öffentliche Ordnung und die Sittlichkeit verletzt ${ }^{10}$. Zu bemerken sei noch, dass die Verordnung Nr. 9 des Obersten Gerichtshofes ${ }^{11}$ zusätzliche Kriterien festlegt, wie die Beglaubigung des ausländischen Urteils durch den brasilianischen Konsul und die entsprechende Übersetzung durch einen brasilianischen vereidigten Übersetzer zu erfolgen hat.

Der Artikel 89 sieht andererseits die Annahmen vor, in denen die Zuständigkeit der brasilianischen Gerichte die aller anderen Länder ausschließt. Somit kann das im Ausland ausgesprochene Urteil unter einer der zwei erwähnten Voraussetzungen in Brasilien nicht anerkannt werden $^{12}$.

Nachfolgend beschäftigen wir uns mit den Kontroversen bezüglich der in der Zivilprozessordnung ausdrücklich erwähnten Annahmen der Ausübung der brasilianischen Gerichtsbarkeit, seien sie konkurrierend und ausschließlich.

\section{a) Wohnsitz des Beklagten in Brasilien}

Der Artikel 88, Abs. I der Zivilprozessordnung bestimmt den Wohnsitz des Beklagten als Kriterium zur Festlegung der brasilianischen Gerichtsbarkeit. Es ist wichtig darauf hinzuweisen, dass der Artikel 12 des Einführungsgesetzes der brasilianischen Gesetzesnormen ebenfalls zur Festlegung der Zuständigkeit der brasilianischen Gerichtsbarkeit und zur Lösung einer bestimmten Streitsache das Kriterium des Wohnsitzes des Beklagten anwendet. Die Artikel 70 bis 78 des Zivilgesetzbuches befassen sich mit dem Sitzkonzept und mit dem Wohnsitzkonzept ${ }^{13}$.

Helio Tornaghi ${ }^{14}$ ist der Meinung, dass der Artikel 94, $\S 3$ der

10 BARBI, Celso Agrícola. Comentários ao Código de Processo Civil.9. Auflage. Rio de Janeiro: Firenze, 1994, Seite 241.

$11 \mathrm{Ab}$ der Verfassungsänderung Nr. 45 vom 2004, sieht der Art. 105, I, Buchstabe i der brasilianischen Verfassung vor, dass das Oberste Gerichtshof (STJ) für die Anerkennung ausländischer Urteile zuständigkeit ist. Bis dahin lag die Zuständigkeit bei dem Brasilianischen Bundesverfassungsgericht (STF).

12 BARBI, Celso Agricola. Comentário ao Código de Processo Civil. 9. Auflage. Rio de Janeiro: Forense, 1994, Seite 243.

13 BARBI, Celso Agrícola. Comentário ao Código de Processo Civil. 9. Auflage. Rio de Janeiro: Forense, 1994, Seite 241.

14 TORNAGHI, Hélio. Comentários ao Código de Processo Civil. Band 1, São Paulo: RT, 1974, Seite 305. 
Zivilprozessordnung eine andere Interpretation des Wortes „Wohnsitz“ des Absatzes I des Artikels 88 gibt. Gemäß Artikel 94, § 3 muss die Klage am Wohnsitz des Klägers erhoben werden, wenn der Beklagte weder wohnhaft noch ansässig in Brasilien ist. Wenn Letzterer ebenfalls nicht in Brasilien ansässig ist, so wird die Klage an irgendeinem Gerichtsstand eingereicht". Helio Tornaghi unterscheidet zwischen Zivil- und Verfahrenswohnsitz und versteht unter dem Letzteren den Ort, wo der Beklagte sich befindet.

So versteht Tornaghi, dass diese Vorschrift das Gericht ermächtigt, die Klage eines in Brasilien wohnhaften Klägers anzuerkennen im Falle, dass dieser der einzige Verbindungsfaktor der Klage zum Lande darstellen sollte. Dies wiederum würde eine weitere konkurrierende Zuständigkeitsannahme der brasilianischen Gerichtsbarkeit darstellen. Carmen Tiburcio stimmt dieser Theorie nicht zu, indem sie meint, dass der Artikel 94, § 3 sich auf das Kapitel der internen Zuständigkeit beziehe und somit in harmonischer Form mit dem Artikel 88 interpretiert werden muss:

wenn in Brasilien eine Pflicht erfüllt werden muss oder wenn die Klage ihren Ursprung in einer in Brasilien erfolgten Handlung oder eines Geschehnisses hat (Artikel 88, II und III) dessen Anerkennungskompetenz bei dem Gericht intern liegt und der Beklagte nicht in Brasilien wohnhaft ist. Die Lösung ist, die Klage bei dem Gerichtsstand des Klägers einzureichen, gemäß Artikel 94, $\S 3^{15}$.

Tornaghi meint ebenfalls, dass die Zivilprozessordnung, in diesem gleichen Artikel 94, § 3, klarstelle, dass das Wohnungskonzept an und für sich genügt, um die Zuständigkeit der brasilianischen Gerichtsbarkeit zu begründen ${ }^{16}$. Barbosa Moreira ist der Ansicht, dass das Wohnsitzkonzept dem „lex fori“" entspreche, d. h. das Konzept der brasilianischen Gesetzgebung ${ }^{17}$.

In Bezug auf den Sitz der juristischen Person sieht der Artikel 88, Einzelparagraph, vor, dass die ausländische juristische Person ihren Sitz in Brasilien hat, wenn sie hier durch Tochterunternehmen, Filiale oder Zweigniederlassungen repräsentiert wird. Dazu äußerte sich Barbosa Moreira dahingehend, dass es nicht notwendig sei, dass der

15 TIBURCIO, Carmen. Temas de Direito Internacional. Rio de Janeiro: Renovar, 2006, Seite 456.

16 TORNAGHI, Hélio. Comentários ao Código de Processo Civil, Band 1, São Paulo: RT, 1974, Seite 305.

17 MOREIRA, José Carlos Barbosa. Probleme in Bezug auf internationale Streitigkeiten. In: Temas de Direito Processual. 5. Serie. Rio de Janeiro: Saraiva 1994, Seite 142. 
Rechtsstreit seine Ursache in einer Handlung der Tochterunternehmen habe, um die Zuständigkeit Brasilianischer Gerichte zu begründen. Es genüge, dass sich eine Agentur, Filiale oder Zweigniederlassung der ausländischen juristischen Person in Brasilien befindet, um in Brasilien verklagt werden zu können. Barbosa Moreira und Barbi ${ }^{18}$ hingegen teilen das Verständnis, dass diese Vorgabe restriktiv interpretiert werden soll, wobei der Einzelparagraph des Artikels 88 nur für Klagen über ausgeführte Handlungen der Agentur, Filiale oder Zweigniederlassung, die in Brasilien ansässig sind, anwendbar ist ${ }^{19}$.

Es stellt sich die Frage, wie die Anwendung des Einzelparagraphs des Artikels 88 sich zu der Theorie der ökonomischen Gruppe verhält. Laut dieser Theorie sind die in verschiedenen Teilen der Erde präsenten Agenturen, Filialen und Zweigniederlassungen Teile einer selben ökonomischen Gruppe und führen ein bestimmtes Muster in Bezug auf die Wirkung der Wirtschaftspolitik aus. Große Unternehmen mit internationaler Wirkung, trotz ihrer bei den Tochtergesellschaften zerstreuten Entscheidungsmacht und juristischer Diversität ${ }^{20}$, besitzen eine ökonomische Einheit die dazu führt, eine Konfusion der Personalitäten oder der Aktivitätswirkungskreise und hauptsächlich eine Konfusion der Vermögen anzunehmen.

In diesem Sinne führt die Herstellung einer juristischen Beziehung mit irgendeiner Unternehmensgesellschaft einer ökonomischen Gruppe mit ausländischer Wirkung zu Zweifeln in Bezug auf die Ausdehnung der Inzidenz des Artikels 88, Einzelparagraph der Zivilprozessordnung. Mit anderen Worten ist es notwendig zu klären, ob zur Ausübung der brasilianischen Gerichtsbarkeit - auf der Grundlage des Einzelparagraphs des Artikels 88 - die juristische Beziehung mit der brasilianischen Agentur, Filiale oder Zweigniederlassung gegeben ist; oder ob es andererseits genügt, wenn die juristische Beziehung zwischen irgendeiner Einheit der ökonomischen Gruppe mit Agenturen, Filialen oder Zweigniederlassungen in Brasilien hergestellt wird.

Über dieses Thema entschied der Oberste Gerichtshof (STJ) in dem Revisionsverfahren Nr. 63.981/SP ${ }^{21}$ in dem Sinne, dass die globalisierte Wirtschaft es ermöglicht, eine Klage bei den brasilianischen Gerichten einzureichen, selbst wenn die juristische Beziehung zwischen dem brasilianischen Verbraucher und einem

18 BARBI, Celso Agrícola. Comentários ao Código de Processo Civil. 9. Auflage. Rio de Janeiro: Forense, 1944, Seiten 241 und 242.

19 MOREIRA, José Carlos Barbosa. Probleme in Bezug auf internationale Streitigkeiten. In Temas de Direito Processual. 5. Serie. Rio de Janeiro: Saraiva, 1994, Seiten 142 und 143.

20 MELLO, Celso D. de Albuquerque. Direito Internacional Econômico. Rio de Janeiro: Renovar, 2003, Seite 104.

21 Oberster Gerichtshof. Revision Nr. 63981/SP, Berichterstatter Minister Aldir Passarinho Junior, 4. Senat. Tagesanzeiger der Justiz vom 20.11.2000. Seite 296. 
ausländischen Unternehmen mit Sitz außerhalb Brasiliens besteht. Und das, weil die wirtschaftliche Gruppe des in Frage gestellten Unternehmens ebenfalls in Brasilien ansässig war und somit die Annahme des Einzelparagraphs des Artikels 88 der Zivilprozessordnung gegeben wäre. Hervorzuheben ist jedoch, dass der angeführte Fall sich auf eine Annahme der Verbraucherbeziehung bezog, für welche die brasilianische Gesetzgebung einen Sonderstatus zum Schutz des Verbrauchers einräumt.

\section{b) Erfüllung der Verpflichtung in Brasilien}

In Bezug auf den Erfüllungsort der Verpflichtung, äußert Pontes de Miranda ${ }^{22}$, dass dieser festgelegt werden kann (a) durch den ausdrücklich oder konkludent geäußerten Willen der Parteien des Rechtsgeschäfts; (b) aufgrund der Verpflichtungsnatur; oder (c) aufgrund des Gesetzes. In diesem Falle meint der Autor, dass der Absatz II des Artikels 88 der Zivilprozessordnung „den Leistungsort als Voraussetzung unabhängig von dem Wohnsitz ausreichen lasse“, sodass ,jedweder Leistungsort - mit Ausnahme eines notwendigen Sondergesetzes („lex specialis“) - je nach dem Willen der Parteien unter Vereinbarung eines anderen geändert werden kann". Auf diese Weise versteht Pontes de Miranda, dass die explizite oder implizite Festlegung des Leistungs- oder Erfüllungsortes für die Vollendung des Rechtsgeschäfts obligatorisch ist.

Barbi erwähnt, dass die im Absatz II des Artikels 88 der Zivilprozessordnung vorgesehene Norm bereits im Artikel 12 des Einführungsgesetzes der brasilianischen Gesetzesnormen ist, und zwar dort wo die Zuständigkeit für diese Annahmen als konkurrierend definiert wird. In diesem Fall, dem Autor nach, ist der Ort an dem die Verpflichtung eingegangen wird unbeachtlich: relevant ist lediglich, dass deren Erfüllung auf brasilianischem Boden erfolgt ${ }^{23}$.

Tornaghi bekräftigt ebenfalls, dass der Ort an den die Verpflichtung eingegangen wurde, unbeachtlich ist.

Damit die brasilianische Gerichtsbarkeit in diesem Falle zuständig wird, ist es notwendig, dass in Brasilien der ,,locus destinatae solutionis “ ist, das heißt, der Ort an dem die Ausführung zu erfolgen hat. Die Freiheit des Schuldners der eine Teilzahlung in Brasilien tätigte obwohl er dazu nicht verpflichtet

22 PONTES DE MIRANDA, Francisco Cavalcanti. Comentários ao Código de Processo Civil, Band II, Rio de Janeiro: Forense, 1973, Seite 190.

23 BARBI, Celso Agricola. Comentários ao Código de Processo Civil. 9. Auflage. Rio de Janeiro: Forense, 1994, Seite 242. 
war, gibt dem Gläubiger nicht die Freiheit, bei brasilianischen Gerichten die Restzahlung zu fordern ${ }^{24}$.

In Bezug auf die Verpflichtungen des Absatzes II des Artikels 88 ist Tornaghi der Meinung, dass es nicht genüge, jegliche Vertragsverpflichtung mit brasilianischem Erfüllungsort zu berücksichtigen. Notwendig ist, dass die Hauptverpflichtung - die der Anlass des Interessenkonfliktes ist - in Brasilien erfüllt werden muss ${ }^{25}$.

\section{c) Klagen, die ihren Ursprung in einem in Brasilien eingetretenen Umstand oder einer in Brasilien vorgenommenen Handlung haben}

Bei der dritten Annahme der konkurrierenden Zuständigkeit brasilianischer Gerichte wird auf jedwedes juristisch relevantes Geschehen Bezug genommen - erlaubt oder unerlaubt, mit oder ohne ausländische Beteiligung - welches als Folge einen Anspruch aufweist ${ }^{26}$.

Laut Celso Agricola Barbi, bezieht sich die Annahme des Artikels 88, Absatz III ,auf eine Tat, die auf einer in Brasilien geschehenen Tatsache beruht. Das heißt, wenn die Klage als Grund diese Tatsache oder Tat hat ${ }^{\text {" } 27}$. Für Helio Tornaghi ist es zum Zwecke des Absatzes III des Artikels 88 einfach notwendig, dass der Grund der Tat auf brasilianischem Boden liegt ${ }^{28}$.

Die brasilianischen Gerichte verfügen über internationale konkurrierende Zuständigkeit für transnationale Schäden, die über das Internet erfolgen, nur in den Fällen, die unter dem Artikel 88 der Zivilprozessordnung vorgeschrieben sind ${ }^{29}$. Somit, wenn der Beklagte

24 TORNAGHI, Helio. Comentários ao Código de Processo Civil. Band 1, São Paulo: RT, 1974, Seite 306.

25 TORNAGHI, Helio. Comentários ao Código de Processo Civil, Band 1, São Paulo: RT, 1974, Seite 306.

26 PONTES DE MIRANDA, Francisco Cavalcanti. Comentários ao Código de Processo Civil, Band II, Rio de Janeiro: Forense, 1973, Seiten 193 und 194. Pontes de Miranda schließt die rechtszeugende?? Tatsachen und Taten strenger Bedeutung, die nicht erlaubten rechtszeugenden Tatsachen und Taten, Taten der unerlaubte, die rechtszeugenden Tatsachen/Taten sowie die Rechtsgeschäfte allesamt in der Gruppe der rechtszeugenden Fakten, Tatsachen und Taten ein. 27 BARBI, Celso Agricola. Comentários ao Código de Processo Civil. 9. Auflage. Rio de Janeiro: Forense, 1994, Seite 242.

28 TORNAGHI, Helio. Comentários ao Código de Processo Civil. Band 1, São Paulo: RT, 1974, Seite 306.

29 ROBERTO, Wilson Furtado. Dano Transnacional e internet: direito aplicável e competência internacional. Curitiba: Juruá, 2010, Seite 117. 
- unabhängig seiner Nationalität - in Brasilien wohnhaft is $\mathrm{t}^{30}$, wird die brasilianische Gerichtsbarkeit zur Lösung des Konflikts zuständig sein, selbst wenn die für den Angriff auf die Ehre des Individuums (Brasilianer oder Ausländer) benutzte Webseite im Ausland betrieben wird $^{31}$.

Die brasilianischen Gerichte verfügen auch über konkurrierende internationale Zuständigkeit, wenn ein gegen das Persönlichkeitsrecht verstoßendes Ereignis oder wenn eine rechtswidrige Tat auf brasilianischem Boden erfolgt ist. Wilson Furtado ist der Meinung, dass ,aus dem Wortlaut der Vorschrift zu entnehmen ist, dass sowohl der Ort der zugrunde liegenden Tat (begangene unerlaubte Tat) als auch die daraus resultierenden Folgen (vollendetes unerlaubtes Geschehnis) die Gerichtsbarkeit festlegen“"32. Ein Problem zeigt sich in Bezug auf die Interpretation des Artikels 88, Absatz III der Zivilprozessordnung: die Tatsache, dass nicht genügend Rechtsprechung vorhanden ist, um das Thema Schadenstatort in Bezug auf den elektronischen Raum entsprechend zu interpretieren.

Der einzige Präzedenzfall auf dem Obersten Gerichtshof (STJ) liegt bei der Analyse der Revision Nr. 1.168.547/RJ. In diesem Fall geht es um die Frage, ob eine natürliche, in Brasilien wohnhafte Person im Falle eines Dienstleistungsvertrages, in dem der Gerichtsstand Spanien ist, das brasilianische Gericht zum Tätigwerden auffordern kann. Hierzu: die Klägerin arbeitete als Tänzerin und Direktionsassistentin einer typisch brasilianischen Show für ein spanisches Unternehmen. Die Vorführungen fanden in Europa und Afrika statt. Monate nach Vertragsabschluss ,besuchte die Klägerin die elektronische Adresse des Unternehmens über das Internet und stellte fest, dass die Seite verschiedene Aufnahmen ihrer Person, entsprechend zusammengestellt aus Veranstaltungen an denen sie teilnahm, zeigte. Einige davon wurden für Werbungszwecke verwendet ${ }^{633}$. Die Autorin reichte mit der Begründung, der Inhalt sei über das Internet weltweit ohne ihre Zustimmung verfügbar eine Klage wegen immaterieller und materieller Schäden ein.

Der Oberste Gerichtshof berücksichtigte, dass die rechtswidrige Tat in Brasilien vorgenommen wurde, gemäß Artikel 88, Absatz III der

30 Einschließlich die einfache Wohnung (Artikel 7, § 8 des Einführungsgesetzes der brasilianischen Gesetzesnormen) oder der Ort an dem sich die Person befindet, falls sie keinen gewöhnlichen Wohnsitz hat (Artikel 73 des Zivilgesetzbuches).

31 ROBERTO, Wilson Furtado. Dano Transnacional e internet: direito aplicável e competência internacional. Curitiba: Juruá, 2010, Seite 105.

32 ROBERTO, Wilson Furtado. Dano Transnacional e internet: direito aplicável e competência internacional.Curitiba: Juruá, 2010, Seite 109.

33 Oberster Gerichtshog, Revision Nr. 1168547/RJ, Berichterstatter Minister Luis Felipe Salomão, Vierter Senat. Tagesanzeiger der Justiz vom 07.02.2011, Seite 4. 
Zivilprozessordnung und legte als Gerichtsstand den Ort, an dem die elektronische Seite zugänglich war, fest:

Wenn die bemängelte unrichtige Tat über das Internet geschieht, unabhängig vom vorgesehenen Gerichtsstand der Dienstleistung, selbst wenn dieser im Ausland ist, ist im Falle eines Konflikts das brasilianische Gericht zuständig, zumal die Klägerin hier ihren Wohnsitz hat und auch von hier aus die Information ausgetragen wurde. Somit wird die Klage unter Berücksichtigung einer in Brasilien durchgeführten Tat typisiert und mit der Annahme des Artikels 88, Absatz III der Zivilprozessordnung begründet ${ }^{34}$.

Das Votum des Berichterstatters berücksichtigte des Weiteren, dass der negative Effekt der rechtswidrigen Tat eine größere Rückwirkung am Ort, an dem die benachteiligten Personen wohnen, bzw. arbeiten, haben wird: ,somit kann die Klage am Gerichtsstand des Handlungs- bzw. Erfolgsortes eingereicht werden, selbst wenn der Beklagte eine juristische Person ist und einen anderweitigen Wohnsitz hat, zumal die negative Auswirkung der rechtswidrigen Tat größtenteils am Ort in dem der Kläger wohnt oder arbeitet geschieht ${ }^{635}$.

Anscheinend ist die Position der brasilianischen Gerichte $\mathrm{zu}$ diesem Fall noch nicht eindeutig, zumal keine Präzedenzfälle vorliegen.

\section{d) In Brasilien gelegene Immobilien}

Die Tatsache, dass lediglich brasilianische Gerichte befugt sind, Prozesse bezüglich in Brasilien gelegener Immobilien $\mathrm{zu}$ entscheiden, stimmt überein mit der allgemeinen Tendenz der souveränen Staaten, diese Beurteilungsmöglichkeit aufgrund der öffentlichen Ordnung lato sensu und der nationalen Sicherheit einzuschränken ${ }^{36}$. Auch bei zwei ausländischen Parteien sind für Klagen bezüglich in Brasilien gelegener Immobilien brasilianische Gerichte zuständig. Diese Regel ist ebenfalls im Artikel 12, § 1 des Einführungsgesetzes der brasilianischen Gesetzesnormen vorgesehen.

Der Meinungsstreit hinsichtlich dieser Anordnung bezieht sich

34 Oberster Gerichtshof, Revision Nr. 1168547/RJ, Berichterstatter: Minister Luis Felipe Salomão, Vierter Senat. Tagesanzeiger der Justiz vom 07.02.2011, Seite 3.

35 Oberster Gerichtshof, Revision Nr. 1168547/RJ, Berichterstatter: Minister Luis Felipe Salomão, Vierter Senat. Tagesanzeiger der Justiz vom 07.02.2011, Seite 11.

36 ARAUJO, Nadia de. Direito Internacional Privado: Teoria e Prática Brasileira. Rio de Janeiro: Renovar, 2008, Seite 246. 
auf die Natur der Klagen bezüglich Immobilien. So ist es kontrovers, ob die Vorgabe sich auf dingliche Klagen über Immobilien einschränkt oder ob sie, andererseits, ebenfalls jedwede Klage dieser Art betrachtet. Erwähnt sei noch, dass die brasilianische Zivilprozessordnung über diese Kontroverse schweigt, anders als die EU-Verordnung Nr. 44/2001 des Rates der Europäischen Union mit der die dinglichen Immobilienklagen sich auf die staatliche Gerichtsbarkeit beschränken ${ }^{37}$.

Pontes de Miranda meint in Bezug auf diese Diskussion, dass die hiermit analysierte Vorschrift auf alle persönliche oder dingliche Immobilienklagen anwendbar ist ${ }^{38}$. Der Jurist fügt hinzu, dass es irrelevant sei, ob es sich um eine Feststellungs-, Gestaltungs- oder Leistungsklage handele.

Celso Agricola Barbi ist der Meinung, dass der Text der Zivilprozessordnung umfassend ist und sich nicht auf dingliche Klagen beschränkt, sondern sich jedweden Klagen widmet, wie jene über Anmietung, Kauf und Verkauf, Anleihen und andere ${ }^{39}$. Barbosa Moreira ist ebenfalls der Meinung, dass die Vorschrift alle Immobilien betreffenden Klagen umfasst ${ }^{40}$.

Tornaghi jedoch ist anderer Meinung: für ihn beziehen sich diese Klagen ausschließlich auf dingliches Recht; es genügt nicht, dass der Streit sich auf jede Art von Immobiliensachen bezieht ${ }^{41}$.

Über diese Debatte äußerte sich bereits das Brasilianische Bundesverfassungsgericht (Supremo Tribunal Federal - STF) ${ }^{42}$, mit der Behauptung, dass die Vorschrift die ausschließliche Zuständigkeit

37 Der Artikel 22.1 der EU-Verordnung Nr. 44/2001 schreibt vor: „Ohne Rücksicht auf den Wohnsitz sind ausschließlich zuständig: (1) für Klagen, welche dingliche Rechte an unbeweglichen Sachen sowie die Miete oder Pacht von unbeweglichen Sachen zum Gegenstand haben, die Gerichte des Mitgliedstaats, in dem die unbewegliche Sache liegend sind. Jedoch sind für Klagen betreffend die Miete oder Pacht unbeweglicher Sachen zum vorübergehenden privaten Gebrauch für höchstens sechs aufeinander folgende Monate auch die Gerichte des Mitgliedstaats zuständig, in dem der Beklagte seinen Wohnsitz hat, sofern es sich bei dem Mieter oder Pächter um eine natürliche Person handelt und der Eigentümer sowie der Mieter oder Pächter ihren Wohnsitz in demselben Mitgliedstaat haben“.

38 PONTES DE MIRANDA, Francisco Cavalcanti. Comentários do Código de Processo Civil. Band II, Rio de Janeiro: Renovar, 2008, Seite 246.

39 BARBI, Celso Agricola. Comentários ao Código de Processo Civil. 9. Auflage. Rio de Janeiro: Forense, 1994, Seite 243.

40 MOREIRA, José Carlos Barbosa. Probleme in Bezug auf internationale Streitigkeiten. In:Temas de Direito Processual. 5. Serie. Rio de Janeiro: Saraiva, 1994, Seite 143.

41 TORNAGHI, Helio. Comentários ao Código de Processo Civil. Band 1, São Paulo: RT, 1974, Seite 308.

42 Brasilianisches Bundesverfassungsgericht, Beschwerde gegen die Entscheidung des Vorsitzenden zum Plenum des Brasilianischen Bundesverfassungsgerichtes bezüglich Beschwerde über ausländisches Urteil Nr. 7101, Berichterstatter Minister Maurício Corrêa (Präsident). Tagesanzeiger der Justiz vom 14.11.03, Seite 12. 
der brasilianischen Gerichte für persönliche oder dingliche Klagen über in Brasilien befindlichen Immobilien vorsieht. Es hat den Anschein, dass dies die überwiegende Meinung der Rechtsprechung ist. Wichtig ist daran zu erinnern, dass die Festlegung der ausschließlichen Zuständigkeit die Bestätigung des ausländischen Urteils nicht ermöglicht, sowie die Wahl des Gerichtsstandes hindert ${ }^{43}$. Dies bedeutet jedoch nicht, dass das ausländische Gericht über die Klage nicht entscheiden darf. Selbst wenn diese Annahme in der ausschließlichen Zuständigkeit eingegliedert ist, hat diese Tatsache keinen Einfluss auf die Zuständigkeit des ausländischen Gerichtes, das heißt, die Regeln der ausschließlichen Zuständigkeit besitzen keine überterritorialen Effekte. Wenn die Möglichkeit besteht, die Klage in einem anderen Land einzureichen und diese dort auch entschieden werden kann, beeinflusst die ausschließliche Zuständigkeit eines brasilianischen Gerichtes keinesfalls die Parteien des Rechtsstreits. .

Ein weiterer wichtiger Punkt dieser Vorschrift ist noch hervorzuheben. Im Hinblick auf die im Wortlaut des Absatzes I des Artikels 89 „Klagen bezüglich Immobilien“ ist die Rechtsprechung der Ansicht, dass im Falle einer im Ausland getroffener vertraglichen Vereinbarung von geschiedenen Eheleute bezüglich der Aufteilung der Güter, der Oberste Gerichtshof diese Vereinbarung anerkennt, auch wenn die Vereinbarung sich auf in Brasilien gelegene Immobilien bezieht ${ }^{44}$. Dies sei durch Artikel 89 Abs. I nicht ausgeschlossen, da der Wortlaut nur Urteile erfasst, nicht aber auch vertragliche Vereinbarungen. Wichtig ist zu bemerken, dass es nur eine Ausnahme zur Anerkennung gibt: entstehende Zweifel in Bezug auf die Gültigkeit der Abmachungen.

\section{e) Nachlass und Teilung der in Brasilien befindlichen Güter}

Der Absatz II des Artikels 89 der Zivilprozessordnung bezieht sich auch auf die Tatsache, dass einige Güter in Brasilien liegen. Für den Nachlass und die Aufteilung der Güter sind brasilianische Gerichte ausschließlich zuständig, selbst dann wenn der Verstorbene Ausländer

43 MOREIRA, José Carlos Barbosa. Probleme in Bezug auf internationale Streitigkeiten. In: Temas de Direito Processual. 5. Serie. Rio de Janeiro: Saraiva, 1994, Seite 141.

44 In diesem Sinne werden folgende Entscheide erwähnt: Oberster Gerichtshof. Angefochtenes ausländisches Urteil Nr. 979, Berichterstatter Minister Fernando Gonçalves, Tagesanzeiger der Justiz vom 29.08.05, Seite 134; Oberster Gerichtshof. Angefochtenes ausländisches Urteil Nr. 3269, Berichterstatter Minister João Otávio de Noronha, Sondersenat. Elektronischer Tagesanzeiger der Justiz vom 22.05.2012 und Oberster Gerichtshof, angefochtenes ausländisches Urteil Nr. 4913. Berichterstatter: Minister João Otávio de Noronha, Sondersenat, Elektronischer Tagesanzeiger der Justiz vom 22.05.2012: alle Entscheide bekräftigen die Stellung des Obersten Gerichtshof über die Bestätigung eines ausländischen Urteils bezüglich eines zwischen geschiedene Eheleute abgeschlossenen Vertrages. 
war und im Ausland lebte.

Nach der herrschenden Lehre bezieht sich Art. 89 Abs. 2 auf Fälle des Nachlasses und der Verfügung von Todes wegen, hinsichtlich beweglichen und unbeweglichen Sachen die sich in Brasilien befinden. In diesem Sinne äußerte sich Pontes de Miranda in dem er der davon ausgeht, dass diese Vorschrift das Ziel hat, Interventionen ausländischer Gerichte bezüglich des Klagen über den Nachlass oder Verfügungen von Todes wegen zu vermeiden, ohne zu überprüfen, ob der Erblasser Ausländer ist oder nicht, auch wenn er seinen Wohnsitz außerhalb Brasiliens hat ${ }^{45}$. Pontes de Miranda äußert sich in dem Sinne, dass der Artikel 89, Absatz II, unter Gütern in Brasilien auch die beweglichen Sachen in Brasilien versteht. Der Jurist schließt in dieser Kategorie auch Aktien von brasilianischen und ausländischen Unternehmen mit Filialen oder Agenturen in Brasilien ein; Wechsel; bei brasilianische Finanzinstitutionen angelegte Gelder, vorausgesetzt dass deren Abhebung nicht ausschließlich von dem Kontoinhaber bei einer ausländischen Filiale oder Agentur gemacht werden kann oder zum ausländischen Banktransfer verfügbar ist ${ }^{46}$.

Celso Agricola Barbi ${ }^{47}$ bezieht sich ebenfalls auf die Vorschrift als lediglich anwendbar hinsichtlich des Nachlasses und Güterteilungen von Todes wegen, in dem er darauf abstellt, dass die Güter des Nachlasses ebenfalls beweglich, lebend oder immobil sein können. Helio Tornaghi ${ }^{48}$ und Barbosa Moreira ${ }^{49}$ folgen der gleichen Orientierung.

Umstritten ist jedoch auf welcher Art von Teilung sich der Gesetzgeber im Absatz II des Artikels 89 bezog, das heißt, geht es lediglich um die Teilung von Todes wegen oder ist auch die Teilung unter Lebenden unter den ausschließlichen Zuständigkeiten der brasilianischen Gerichte mit inbegriffen.

Die obersten Gerichte äußerten sich bereits über das Thema. Anfänglich, laut Carmen Tiburcio ${ }^{50}$, interpretierte das Brasilianische Bundesverfassungsgericht (STF) die besagte Vorschrift ebenfalls mit dem Einschluss der Teilung der Güter unter Lebenden in Brasilien

45 PONTES DE MIRANDA, Francisco Cavalcanti. Comentários ao Código de Processo Civil, Band II, Rio de Janeiro: Forense, 1973, Seite 195.

46 PONTES DE MIRANDA, Francisco Cavalcanti. Comentários ao Código de Processo Civil, Band II, Rio de Janeiro: Forense, 1973, Seite 195 bis 196.

47 BARBI, Celso Agricola. Comentários ao Código de Processo Civil, 9. Auflage, Rio de Janeiro: Forense, 1994, Seite 243.

48 TORNAGHI, Helio. Comentários ao Código de Processo Civil, Band 1, São Paulo: RT, 1974, Seiten 308 und 309.

49 MOREIRA, José Carlos Barbosa. Probleme in Bezug auf internationale Streitigkeiten. In Temas de Direito Processual. 5. Serie. Rio de Janeiro: Saraiva, 1994, Seite 143 und 144.

50 TIBURCIO, Carmen. Temas de Direito Internacional. Rio de Janeiro: Renovar, 2006, Seite 453. 
und verweigerte somit die Bestätigung verschiedener ausländischer Scheidungsurteile, welche die Teilung von Immobilien in Brasilien zum Gegenstand hatten. Jedoch zu Beginn der 80er Jahre ${ }^{51}$, änderte das Brasilianische Bundesverfassungsgericht (STF) seine Einstellung und ließ zu, dass die Vorgabe ausschließlich für Bestandsaufnahmen und Teilungen von Todes wegen Geltung hatte. Laut der Autorin war dies das vorwiegende Verständnis des Obersten Gerichtshof (STJ). Neuerdings jedoch, mit der Revision Nr. 275.985/SP ${ }^{52}$, kam der Oberste Gerichtshof (STJ) zur Schlussfolgerung, dass brasilianische Gerichte für die Durchführung der Nachlass und Teilung von in Brasilien befindlichen Gütern zuständig sind (sei es unter Lebenden oder von Todes wegen).

Unter Berücksichtigung, in jeglicher Form, des mehrheitlichen Verständnisses der Rechtslehre, dass der Artikel 89, Absatz II sich nur auf die Bestandsaufnahme und Teilung von Tod wegen bezieht, ist die Behandlung des Themas der Teilung unter Lebenden fraglich.

In diesem Sinne, im Falle einer Teilung von beweglichen Gütern im Ausland und Immobilien in Brasilien, wird die ausschließliche Zuständigkeit der brasilianischen Gerichtsbarkeit in Frage gestellt. Kraft des Artikels 89, Absatz I, würde auch die Zuständigkeit zur Entscheidung der beweglichen Güter im Ausland hinzugezogen. Alternativ stellt sich die Frage, ob eine Zusammenlegung der Zuständigkeiten, das heißt, der brasilianische Richter würde aufgrund des Artikels 89, Absatz I, über Immobilien entscheiden und der brasilianische oder ausländische Richter würde - ununterscheidbar über die beweglichen Güter im Ausland unterscheiden. Somit ergäbe sich die Annahme der konkurrierenden Zuständigkeit ${ }^{53}$. Der Oberste Gerichtshof (STJ) besitzt jedoch einen Präzedenzfall in dem Sinne, dass die von der ausländischen Gerichtsbarkeit durchgeführte Teilung

51 In diesem Zusammenhang, siehe Brasilianisches Bundesverfassungsgericht, Beschwerde gegen Entscheidung des Vorsitzenden an Kammerplenum über ausländisches Urteil Nr. 2492. In: Revista Trimestral de Jurisprudência, Band 101, 1982, Seite 69.

52 Oberster Gerichtshof. Revision Nr. 275.985/SP, Berichterstatter Minister Sálvio de Figueiredo Teixeira. Tagesanzeiger der Justiz vom 13.10.03, Seite 366.

53 Das Brasilianische Bundesverfassungsgericht behandelte bereits das Thema. Differenzierte jedoch nicht die Gütertypen - mobile oder immobile - die sich im Ausland befanden. Im erwähnten Fall verstand das Brasilianische Bundesverfassungsgericht, dass im Falle einer Scheidungsteilung - mit Güter sowohl in Brasilien als auch im Ausland - läge die Kompetenz bei den Angehörigkeitsstaaten beider Eheleute, in denen sie wohnhaft und ansässig wären. Konsequenterweise entschied sich das Brasilianische Bundesverfassungsgericht für die Anerkennung des ausländischen Urteils über die Teilung der Güter des Paares, selbst wenn die Güter in Brasilien sich befänden. (Brasilianisches Bundesverfassungsgericht. Beschwerde gegen Entscheidung des Vorsitzenden an Kammerplenum: ausländisches Urteil Nr. 2396, Berichterstatter Minister Thompson Flores, Plenum, Tagesanzeiger der Justiz vom 28.12.1978, Seite 10573). 
aufgrund einer Scheidung in Brasilien anerkannt werden kann, selbst wenn Immobilien zu teilen sind. Diese Handlungsweise erscheint uns nicht als die zutreffendste Alternative.

In einer ausschließlichen Teilung von ausländischen Immobilien erscheint uns die bilaterale Anwendung des Artikels 89, Absatz I nicht geeignet für die Ablehnung der Gerichtsbarkeit der brasilianischen Gerichtsbarkeit. Es ist jedoch notwendig, dass die brasilianische Gerichtshoheit die zukünftige Auswirkung ihrer Entscheidung überdenkt, hauptsächlich vor dem Hintergrund, dass es eine Tendenz der Gerichtsverordnungen diverser Länder ist, ihre Zuständigkeiten für Klagen über Immobilien an die Gerichtsstände der jeweiligen Immobilien zu übertragen.

Es hat den Anschein, dass in einem Scheidungsprozess mit der Teilung von lediglich beweglichen Gütern - sei es in Brasilien oder im Ausland - die Gerichtsbarkeit sowohl von der brasilianischen als auch von der ausländischen Gerichtshoheit ausgeübt werden kann. Dies ergibt, wie bereits erwähnt, die Annahme der konkurrierenden Zuständigkeit. Damit ist die Annahme möglich, dass die Aufzählung der Fälle der internationalen Gerichtsbarkeit der Zivilprozessordnung beispielhaft ist und, dass auch andere Umstände existieren, unter denen die brasilianische Gerichtsbarkeit ausgeübt werden darf.

\subsection{Annahmen der nicht ausdrücklich in der Zivilprozessordnung vorgesehenen Ausübung der brasilianischen Gerichtsbarkeit}

In der Rechtslehre und in der Rechtsprechung wird über die Existenz anderer Annahmen zur Ausübung der brasilianischen Gerichtsbarkeit - außer der in den Artikeln 88 und 89 der Zivilprozessordnung erwähnten Fällen - dahingehend diskutiert, ob diese Vorschriften eine abschließende oder beispielhafte Liste darstellt.

Celso Agricola Barbi vertritt die Meinung, dass der Staat eine direkte Abgrenzung seiner Gerichtsbarkeit festlegen kann, indem er die ihm unterliegenden Fälle ausdrücklich benennt und, implizit, die restlichen ausschlie $3 \mathrm{t}^{54}$. In Bezug auf die Artikel 88 bis 90 ist Barbi der Ansicht, dass ,direkt" festgelegt wurde, ,in welchen Fällen die brasilianische Gerichtsbarkeit zuständig ist" ${ }^{\text {"55 }}$. Auf dieser Weise hat es den Anschein, dass der Autor die vorgegebene Aufstellung als abschließend für die Ausübungsannahmen der brasilianischen Gerichtsbarkeit hält.

54 BARBI, Celso Agricola. Comentários ao Código de Processo Civil. 9. Auflage. Rio de Janeiro: Forense, 1994, Seite 240.

55 BARBI, Celso Agricola. Comentários ao Código de Processo Civil. 9. Auflage. Rio de Janeiro: Forense, 1994, Seite 240. 
Anderer Meinung ist Helio Tornagui ${ }^{56}$, in dem er meint, dass der Artikel 94, § 3 der Zivilprozessordnung eine weitere Annahme der internationalen Zuständigkeit aufführt und somit $\mathrm{zu}$ verstehen ist, dass die dargestellten Fälle der Artikel 88 und 89 nur beispielhaft sind. Pontes de Miranda hat das Verständnis, dass „die Vorschriften lediglich das Thema der Zuständigkeit der brasilianischen Gerichte ab betreffen. Die Artikel 88 bis 90 führen nur die Klagearten auf ${ }^{657}$, was den beispielhaften Charakter der besagten Vorschriften unterstreiche. verstärkt.

José Carlos Barbosa Moreira ${ }^{58}$ vertritt die Meinung, dass die aufgelisteten Fälle in den Artikeln 88 und 89 zur Festsetzung der Voraussetzungen, unter denen die brasilianische Justiz kompetent zur Lösung eines bestimmten Streits ist, nicht abschließend sind und somit nur eine beispielhafte Aufstellung darstellen. Um seine Meinung zu erläutern meint Barbosa Moreira, dass die Zivilprozessordnung sich nicht auf Fälle der freiwilligen Gerichtsbarkeit bezieht, das heißt, in solchen Fällen in denen es keinen Beklagten und keine Klage gibt, wie bei einvernehmlicher Trennung der Ehegatten, der Gütereinnahme von Verschollenen, die Betreuung von Geisteskranken, und anderen Fällen.

In diesem Sinne ist der Autor der Ansicht, dass die Gesetzeslücke in dieser Angelegenheit mit der Anwendung unserer internen Zuständigkeitsregeln - die Rechtsvorschrift, die das zuständige brasilianische Gerichtsorgan zur Entscheidung einer Rechtssache definiert - auch die internationale Zuständigkeit zweckhalber festlegen solle. In Bezug, jedoch, auf die freiwillige Gerichtsbarkeit ist keine ausdrückliche Zuständigkeitsregel in der Gesetzgebung zu finden. Somit, auch wenn nicht vollkommen zufriedenstellend, sollten die Analogie und die allgemeinen Rechtsprinzipien Anwendung finden. Haroldo Valladão ist ebenfalls dieser Meinung ${ }^{59}$.

Botelho de Mesquita meint ebenfalls, dass die in der Vorschrift aufgeführten Annahmen beispielhaften Charakter besitzen, weil einige Prinzipien des internationalen Rechtes, wie das Prinzip der Vermeidung der Rechtsverweigerung und das Effektivitätsprinzip, einzuhalten sind. Deshalb sind neue Anwendungsannahmen der Artikel 88 und 89 der Zivilprozessordnung möglich ${ }^{60}$.

56 TORNAGHI, Helio. Comentários ao Código de Processo Civil. Band I. São Paulo: RT, 1974 , Seite 305

57 PONTES DE MIRANDA, Francisco Cavalcanti. Comentários ao Código de Processo Civil. Band II, Rio de Janeiro: Forense, 1973, Seite 193.

58 MOREIRA, José Carlos Barbosa. Probleme in Bezug auf internationale Streitigkeiten. In Temas de Direito Processual. 5. Serie. Rio de Janeiro: Saraiva, 1994, Seite 144.

59 VALLADÃO, Haroldo, Direito Internacional Privado, Band III, Rio de Janeiro: Freitas Bastos, 1978, Seiten 132, 133, 134 und 137.

60 MESQUITA, José Ignácio Botelho de. Über die internationale Kompetenz und ihre 
Für Leonardo Greco ist die Auflistung des Artikels 88 der Zivilprozessordnung ebenfalls nicht abschließend und somit kann praktisch jedes Klageverfahren gegenüber brasilianischen Gerichten eingereicht werden, nur wenn das Effektivitätsprinzip eingehalten wird. Dem Autor zufolge: ,wenn das Effektivitätsprinzip nicht gefährdet wird, kann das Verfahren der brasilianischen Justiz vorgetragen werden, selbst wenn keine der Annahmen der Absätze I bis III des Artikels 88 vorhanden sind" ${ }^{\text {"61 }}$.

Antenor Madruga ${ }^{62}$ ermittelt, dass die brasilianischen Obergerichte bereits die Möglichkeit hatten, sich über weitere internationale Zuständigkeitsannahmen - die nicht in der jetzigen Zivilprozessordnung aufgeführt werden - zu äußern. Beispiel zu dieser Bemerkung ist der Beschwerde Nr. 64, mit dem der Oberste Gerichtshof (STJ) vorgab, dass ,die internationale Zuständigkeit (Gerichtsbarkeit) der brasilianischen Gerichtshoheit sich nicht nur in der Analyse der Artikel 88 und 89 der Zivilprozessordnung - dessen Inhalt nicht abschließend ist - erschöpft ${ }^{63}$.

\subsection{Geplante Änderungen hinsichtlich der Ausübung der brasilianischen Gerichtsbarkeit im Projekt der Zivilprozessordnung}

Die letzte Version des Projektes der Zivilprozessordnung versuchte die technische Ungenauigkeit der Nomenklatur,,internationale Zuständigkeit" zu korrigieren, in dem sie Vorschriften über das Thema im Kapitel „Grenzen der nationalen Gerichtsbarkeit“ einfügte. Die Vorschriften haben insgesamt dien Bezug zu der brasilianischen Gerichtsbarkeit beibehalten.

Die Ausarbeitung des Gesetzesvorhabens unterteilt die Zuständigkeitsregelungen in drei Vorschriften. Der erste Artikel behält den Wortlaut des aktuellen Artikels $88 C P C$ bei. Um zu verdeutlichen, dass die drei vorgesehenen Zuständigkeitsregelungen nicht kumulativ zu verstehen sind, hat der Gesetzgeber die Verbindung "oder, zwischen den einzelnen Vorschriften eingefügt. Darüber hinaus bezieht sich der dritte Absatz ausdrücklich auf einen Umstand oder eine in Brasilien

Informationsprinzipien. In Revista do Processo 50-51, 1988, Seite 56.

61 GRECO, Leonardo. Die internationale Kompetenz der brasilianischen Justiz. In Revista da Faculdade de Direito de Campos, Jahr VI, Nr. 7, Dezember 2005, Seiten 180 und 181.

62 MADRUGA, Antenor. Homologação de sentença estrangeira de falência.Verfügbar in: http://www.conjur. com.br/2011-jun-22/judiciario-estrangeiro-nao-decretar-falencia-empresabrasileira. Abgerufen am 23.01.2013.

63 Oberster Gerichtshof. Beschwerde Nr. 64/SP, Berichterstatterin Ministerin Nancy Andrighi, dritter Senat. Elektronischer Tagesanzeiger der Justiz vom 23.06.2008. Im gleichen Sinne siehe Brasilianisches Bundesverfassungsgericht. Rechtshilfeersuchschreiben Nr. 9697, Berichterstatter Minister Carlos Velloso (Präsident). Tagesanzeiger der Justiz vom 24.04.2001. 
vorgenommene Handlung als Klagegrund ${ }^{64}$.

Danach fügte der Gesetzgeber im Projekt eine zweite Vorschrift ein, dessen „Caput" identisch mit dem Vorhergehenden ist. Darin wurden drei Annahmen der brasilianischen Gerichtsbarkeit hinzugefügt ${ }^{65}$. Die erste Annahme dieser Vorschrift bezieht sich auf Unterhaltsklagen wenn (a) der Kläger in Brasilien wohnhaft oder ansässig ist; oder (b) der Beklagte Verbindungen zu Brasilien unterhält wie Besitz oder Eigentum von Gütern, Einkünfte oder Empfang von finanziellen Begünstigungen. Für die Unterhaltsklagen wurde also die Möglichkeit eingeräumt, die Gerichtsbarkeit aufgrund (a) des Wohnsitzes des Klägers (,forum actoris“) oder (b) der Existenz von Vermögen am Gerichtsstand (,forum patrimonii“") auszuüben.

Die zweite in das Projekt eingefügte Annahme der Vorschrift bezieht sich auf Verbraucherbeziehungen, also wenn der Verbraucher in Brasilien wohnhaft ist. Die Norm trägt eine weitere Annahme der Gerichtsbarkeitsausübung in Bezug auf den Wohnsitz des Autors vor mit der wahrscheinlichen Absicht die Wirksamkeit des Verbraucherrechtes in der brasilianischen Gesetzgebung zu gewährleisten. Diese ist Teil der öffentlichen Ordnung. Aus der Unternehmensperspektive unterwirft die Vorschrift potenziell jedes Unternehmen der brasilianischen Gerichtsbarkeit. Als Beispiel nimmt man einen durch einen Verbraucher getätigten Kauf im Ausland von einem Unternehmen, das keinerlei Kontaktstellen in Brasilien hat; es genügt, dass der Verbraucher in Brasilien wohnhaft oder ansässig ist, damit die brasilianische Gerichtsbarkeit hergestellt wird.

Die dritte von dem Gesetzgeber eingeführte Annahme bezieht sich auf die ausdrückliche oder stillschweigende Unterwerfung der Parteien der brasilianischen Gerichtsbarkeit. Die Vorschrift legt die Möglichkeit fest, die brasilianische Gerichtsbarkeit aufgrund der Willensautonomie auszuwählen, indem sie die Wahl des brasilianischen Gerichtsstandes zulässt. Es ist anzumerken, dass der Gesetzgeber andere Situationen, die nicht in der vorhergehenden Ordnung vorgesehen

64 Es ist Aufgabe der brasilianischen Gerichtshoheit die Bearbeitung und Entscheidung der Klagen in denen: I - der Angeklagte - unabhängig seiner Staatsangehörigkeit - in Brasilien wohnhaft ist; II - de Schuld in Brasilien zu verbüßen ist; oder III - die begründete Tat oder Tatsache in Brasilien geschehen ist. Einzelparagraph - Zum Zwecke des Absatzes I ist vorauszugehen, dass die ausländische juristische Person hier. zu Lande eine Agentur, Filiale oder Zweigniederlassung besitzt.

65 Des Weiteren ist es Aufgabe der brasilianischen Gerichtshoheit die Bearbeitung und Entscheidung der Klagen über: I - Alimente, wenn a) der Kläger in Brasilien wohnhaft oder ansässig ist; oder b) der Angeklagte Verbindungen zu Brasilien unterhält wie Besitz oder Eigentum von Gütern, Einkünfte oder Empfang von finanziellen Begünstigungen; II Konsumbeziehungen, wenn der Verbraucher in Brasilien wohnhaft oder ansässig ist; III - Fälle bei denen die Parteien - formell oder informell - der brasilianischen Gerichtsbarkeit unterliegen. 
waren herstellt. In diesen darf die brasilianische Gerichtshoheit ihre Gerichtsbarkeit ausüben. In gleicher Weise ist zu beobachten, dass die Annahme sehr weit ausgelegt wird und erlaubt, dass ausländische Parteien in Brasilien Prozesse führen können, selbst wenn sie keinerlei Verbindungselemente mit dem Gerichtsstand haben.

Es ist darauf hinzuweisen, dass der Gesetzgeber nicht klarstellt, ob die drei in der Vorschrift eingefügten Annahmen des Projektes den Umständen der ausschließlichen oder konkurrierenden Ausübung der brasilianischen Gerichtsbarkeit entsprechen. Es besteht die Möglichkeit der Behauptung, aufgrund des identischen Gesetztextes mit dem ersten Artikel, dass die Vorschrift ebenfalls Annahmen zur konkurrierenden Zuständigkeit darstellt. Jedoch sind bei dieser Annahme einige Ungewissheiten implizit.

In Bezug auf den Vorschlag der Unterhaltsklagen - dessen Ziel die Einfachheit und Beschleunigung der Alimente ist - wäre die Annahme einer ausschließlichen Gerichtsbarkeit ein Hindernis zur Klage eines im Ausland ansässigen Klägers gegenüber einem in Brasilien ansässigen Beklagten. Es bestünde ein Konflikt mit dem Absatz I des vorhergehenden Artikels, der dem aktuellen Artikel 88, Absatz I der Zivilprozessordnung entspricht. Da es sich außerdem um ausschließliche Zuständigkeit handelt, wäre es dem Kläger unmöglich, eine Klage im Ausland - dort wo der Beklagte wohnhaft ist - zwecks Beschleunigung der Ladung einzureichen, um dann im Nachhinein die Vollstreckung des Urteils in Brasilien zu erwarten. Es hat aber den Anschein, dass es sich um konkurrierende Zuständigkeit handelt, damit der Kläger der Unterhaltsklage so effizient wie möglich sein Ziel erreichen kann.

Bei Verbraucherklagen andererseits würde die Annahme, dass sie der konkurrierenden Zuständigkeit unterliegen, dazu führen, dass ein ausländisches Urteil in Brasilien vollstreckt würde. Dies liefe dem Zweck, den brasilianischen Verbraucher zu schützen, zuwider. Dieses Verständnis würde allerdings erlauben, dass der Verbraucher einen Prozess im Ausland führen würde, falls ihm diese Alternative besser erschiene.

In Bezug auf die Annahme der Unterwerfung der Parteien unter die brasilianische Gerichtsbarkeit würde dies bedeuten, dass konkurrierende Zuständigkeit die Folge wäre, das heißt, es wäre erlaubt, dass das Urteil eines im Ausland ergangenen Urteils in Brasilien vollstreckt würde. Dies ergäbe also eine Nichtbeachtung der Parteien in Bezug auf die Wahl des brasilianischen Gerichtsstandes und würde die Nichtanerkennung des ausländischen Urteils ermöglichen, zumal die ausländische Gerichtshoheit unzuständig wäre. Andererseits würde die Anerkennung der Annahme der ausschließlichen Zuständigkeit die Wahlmöglichkeit eines anderen Gerichtsstandes durch die Parteien 
ausschließen.

Der dritte Artikel des Projektes widmet sich dem Thema der Ausübung der brasilianischen Gerichtsbarkeit und gibt, in seiner Substanz, die Redaktion des Artikels 89 der Zivilprozessordnung wieder. Er nennt ausdrücklich zwei Fälle der ausschließlichen Gerichtsbarkeit ${ }^{66}$. Der erste Absatz wurde wortwörtlich aus der aktuellen Redaktion des Absatzes I des Artikels 89 übernommen. Im Absatz II wurde jedoch der Satz ,in Sachen der Erbfolgen“ hinzugefügt und damit die ausschließliche Zuständigkeitder brasilianischen Hoheit zur Bestandsaufnahme und Güterteilung von Todes wegen klargestellt.

\section{WAHL DES GERICHTSSTANDES}

Bei Streitigkeiten mit ausländischem Bezug ist es üblich, dass die Parteien den für sie günstigsten Gerichtsstand wählen. In diesem Sinne, kann die Wahl des Gerichtsstandes die Ausübung der Gerichtsbarkeit des Staates sowohl ausdehnen als auch ersetzen ${ }^{67}$.

Im brasilianischen Recht sieht der Artikel 111 der Zivilprozessordnung die Möglichkeit derWahldes Gerichtsstandes durch die Parteien vor ${ }^{68}$. Es ist jedoch hervorzuheben, dass dieser Vorschlag sich ausschließlich auf die Annahme der Wahl des Gerichtsstandes im nationalen Bereich bezieht; es gibt somit auf dieser Weise keine ausdrückliche Bestimmung in der brasilianischen Gesetzgebung in Bezug auf die Wahl des Gerichtsstandes auf internationaler Ebene.

Aufgrund dieser Unvorhersehbarkeit wurde die Annahme einer Klausel über die Wahl des Gerichtsstandes im brasilianischen Recht polemisiert. Und dies, weil es fraglich ist, ob der Wille der Parteien die Normen über die Festlegung der Zuständigkeit der brasilianischen Gerichtshoheit der Zivilprozessordnung verändern könnte, zumal es Normen des öffentlichen Rechts sind und direkt aus der Bundesgerichtshoheit fließen ${ }^{69}$. Somit wird die Möglichkeit

66 Es obliegt der brasilianischen Gerichtsbarkeit, unter Ausschluss jedweder anderen: I Die Anerkennung von Klagen über in Brasilien befindlichen Immobilien; II - In Sachen der Erbfolgen, die Durchführung der Bestandsaufnahme und Teilung der in Brasilien befindlichen Gütern, auch wenn der Autor des Nachlasses Ausländer oder im Ausland wohnhaft war.

67 Im französischen Recht, zum Beispiel, wird die Möglichkeit der Ernennung eines Gerichtsstandes zur Ausübung der Gerichtsbarkeit mit der gewählten Nomenklatur „clause attributive de juridiction" evident.

68 Artikel 111. Die Kompetenz bezüglich des Gegenstandes und der Hierarchie ist durch Konvention der Parteien unübertragbar; diese können jedoch die Kompetenz aufgrund des Wertes und des Territoriums modifizieren, in dem sie den Gerichtsstand wählen, an dem die Aktion über Rechte und Pflichten eingereicht wird. § 1 - Die Vereinbarung wirkt jedoch nur wenn vertraglich und formell festgehalten und sich auf einen einzigen Gerichtsfall bezieht. $\S$ 2 - Der vertragliche Gerichtsstand verpflichtet die Erben und Nachfolger der Parteien.

69 TIBURCIO, Carmen. Temas de Direito Internacional. Rio de Janeiro: Renovar, 2006, Seite 
in Frage gestellt, ob es erlaubt wäre, dass die Willensautonomie die Aussagen der brasilianischen Prozessordnung überragen könnte. Auf dieser Weise könnten die Parteien ihren Prozess bei der brasilianischen Justiz führen, selbst wenn dies nicht konkret in den Artikeln 88 und 89 der Zivilprozessordnung vorgesehen ist $^{70}$. Andererseits könnte die Wahl eines nicht brasilianischen Gerichtsstandes zur Lösung der in den Artikeln 88 und 89 der Zivilprozessordnung erwähnten Angelegenheiten zur Folge haben, dass die Ausübung der brasilianischen Gerichtsbarkeit vermieden würde. Im Prinzip ist die Ausübung der Gerichtsbarkeit von staatlichem Interesse.

Aus der Sicht von Carmen Tiburcio würden im Falle, dass die Parteien frei unter sich den Gerichtsstand gemäß den Vorgaben des Artikels 88 der Zivilprozessordnung wählen, die Souveränität des Staates und die öffentliche Ordnung nicht verletzt sein, weil diese Verordnung die Voraussetzung der konkurrierenden Zuständigkeit der brasilianischen Gerichtshoheit vorsieht. Wenn jedoch die Auswahl eines anderen Gerichtsstandes - gemäß der im Artikel 89 aufgelisteten Zuständigkeiten - vereinbart würde, so wäre diese Klausel ungültig, zumal sie damit den Staat von der Ausübung der Gerichtsbarkeit ausschließen würde ${ }^{71}$.

Im Hinblick, dass die herrschende Lehre die Wahl des Gerichtsstandes zulässt ${ }^{72}$, sind wir der Meinung, dass die diesbezüglich uneinheitliche Rechtsprechung zu erwähnen ist.

Im Jahre 1957 äußerte sich das Brasilianische Bundesverfassungsgericht(STF) zum ersten Mal zu dem Thema ${ }^{73}$. Es handelte sich um eine Schadensersatzklage die auf einer Nichterfüllung der sich aus einem Transportvertrag ergebenden, in Brasilien zu erfüllenden, Pflichten beruht, wobei die Parteien Montevideo als Gerichtsstand fürdenFallvonmöglicherweiseauftretendenStreitigkeiten aus dem Vertrag wählten. Das Brasilianische Bundesverfassungsgericht

85.

70 TIBURCIO, Carmen. Temas de Direito Internacional. Rio de Janeiro: Renovar, 2006, Seite 86.

71 TIBURCIO, Carmen. Temas de Direito Internacional. Rio de Janeiro: Renovar, 2006, Seite 87.

72 In diesem Sinne, siehe: TORNAGHI, Helio. Comentários ao Código de Processo Civil, Band I, São Paulo: RT, 1974, Seite 307; MOREIRA, José Carlos Barbosa. Probleme in Bezug auf internationale Streitigkeiten. In Temas de Direito Processual. 5. Serie. Rio de Janeiro: Saraiva, 1994, Seite 146; MESQUITA, José Ignácio Botelho de. Über die internationale Kompetenz und ihre Informationsprinzipien. In Revista do Processo 50-51, 1988, Seite 57; STRENGER, Irineu. In: Contratos Internacionais do Comércio, 2. Auflage, São Paulo: Editora Revista dos Tribunais, 1992, Seiten 256 bis 258.

73 Brasilianisches Bundesverfassungsgericht, Beschwerde Nr. 30636. Berichterstatter Minister Cândido Motta, erster Senat, Entscheid vom 29.05.1957. Zusammenfassung: Band 00298-01, Seite 393. 
(STF) war der Ansicht, dass gemäß Artikel 12 des Einführungsgesetz der brasilianischen Gesetzesnormen ${ }^{74}$, mit Ausnahme der Vorschriften seines 1. Paragraphen ${ }^{75}$, die Wahl ad libitum von den Parteien durch formelle Vereinbarung geändert werden könne, zumal der Inhalt der besagten Vorschrift nicht dem öffentlichen Recht unterliege und die in ihr beinhalteten Norm nicht der brasilianischen Staatshoheit obliege.

1989 lehnte der Oberste Gerichtshof (STJ) die Wahlklausel des Gerichtsstandes nach den Voraussetzungen des Artikels $88 \mathrm{ab}^{76}$. Laut des Justizgerichtes darf in Seetransportverträgen, in denen die Warenausschiffung in Brasilien vorgesehen ist, der von den Parteien gewählte vertraglichen Gerichtsstand nicht geltend gemacht werden, im Hinblick auf dem Vorrang des Absatzes II des Artikels 88 der Zivilprozessordnung. Der Oberste Gerichtshof (STJ) argumentierte auch mit dem Prinzip der Unterwerfung, zumal die unterliegende Partei der ursprünglichen Klage sich erst nach der Niederlage an die Gerichtsstandesklausel erinnerte. In diesem Falle verstand das Gericht, dass es eine stillschweigende Änderung des Gerichtsstandes ab dem Moment gab, als die Lösung des Streits anfänglich in Brasilien - ohne jedwede Klausel der Zuständigkeitsänderung - passierte.

Eine weitere Verneinung über die Anwendung der Gerichtsstandwahlklausel durch der Oberste Gerichtshof (STJ) geschah im Jahre $2000^{77}$. Imbesagten Fall schloss dieBrasoilmitdreiUnternehmen einen Vertrag ab zur Umwandlung eines Erdöltransportschiffes in einer schwimmenden Plattform (Festlandsockel). Die Brasoil forderte zur Durchführung des Vertrages als Garantie, dass zwei nordamerikanische Versicherungsgesellschafteneinenperformancebond-Vertragmitdendrei Unternehmen abschließen sollten. Der Wert dieses Vertrages entsprach dem Wert des Schiffumwandlungsvertrages und hatte als Gerichtsstand New York. Anschließend, unter Angabe der Nichtberücksichtigung des Vertrages, reichte die Brasoil in Rio de Janeiro eine Klage über Schaden- und Klage auf Schadensersatz kumuliert mit der Eintreibung des Versicherungsscheins gegenüber der Vertragsunternehmen und der Versicherungsgesellschaften mit der Aussicht, letztere würden den performance bond bezahlen ${ }^{78}$. Das Justizgericht äußerte ausdrücklich,

74 Artikel 12. Die brasilianische Gerichtshoheit ist kompetent wenn der Angeklagte in Brasilien wohnhaft ist oder wenn die auferlegte Tat hier durchzuführen ist.

75 Artikel 12, § 1: Nur der brasilianischen Gerichtshoheit obliegt die Anerkennung der Klagen die Immobilien in Brasilien betreffen.

76 Oberster Gerichtshof. Wiederaufnahmeklage Nr. 133/RS. Berichterstatter Minister Claudio Santos, Revisor Minister Sálvio de Figueiredo Teixeira. RT Band 656, Seite 180.

77 Oberster Gerichtshof, Revision Nr. 251.438/RJ, vierter Senat, Berichterstatter Minister Barros Monteiro, DJU vom 02.10.2000, Seite 173.

78 TIBURCIO, Carmen. Temas de Direito Internacional. Rio de Janeiro: Renovar, 2006. Seiten 84 und 85 . 
dass die konkurrierende Zuständigkeit des brasilianischen Richters nicht auf den Willen der Parteien reduziert werden darf und erkannte die Überlegenheit der Gerichtsstandwahl-Klausel nicht an. Obwohl es uns unrichtig vorkommt, entschied das Justizgericht treffend mit der Nichtanerkennung der besagten Klausel, zumal im Vertrag zwischen den Versicherungsgesellschaften und den für die Durchführung der Arbeiten verantwortlichen Unternehmen der Gerichtsstand New York festgelegt wurde. Es handelt sich daher um einen untergeordneten Vertrag des Hauptvertrages, in welchen die Klägerin nicht als Partei erscheint. Der Gerichtsstand in den Vereinigten Staaten kann also nicht über die im Artikel 88, Absatz II der Zivilprozessordnung aufgeführte Zuständigkeiten vorwiegend sein. Die Klausel, die für die Veränderung der Zuständigkeit der brasilianischen Judikative verantwortlich ist, gehört zu einem untergeordneten Vertrag des Hauptvertrages, in dem die Brasoil keine der Parteien ist.

$\mathrm{Zu}$ einem späteren Zeitpunkt erkannte der Oberste Gerichtshof (STJ) die Gültigkeit der Klausel an: in einem Fall bezüglich des Verkaufs und Kaufs einer Immobilie in Miami, in den Vereinigten Staaten Nordamerikas ${ }^{79}$. Laut Justizgericht hat der Artikel 88 der Zivilprozessordnung nicht genügend Macht über die Grundsätze bezüglich der Gerichtsstandwahl und über die Tatsache, dass der Autor nicht über einen festen Wohnsitz in Brasilien verfügt. Schließlich wurde argumentiert, dass der Vertrag im Ausland geschlossen wurde, an der Stelle wo sich die betreffende Immobilie befand.

Der Oberste Gerichtshof (STJ) stimmte auch der Gerichtsstandwahlklausel im Jahre $2005 \mathrm{zu}^{80}$, mit der Behauptung, dass die Gerichtsstandwahl gültig ist, vorausgesetzt die Nichttangierung des öffentlichen Interesses. Das selbe Gericht urteilte jedoch im Jahre 2008 zugunsten einer Nichtanerkennung der Gerichtsstandwahlklausel und erwähnte dabei vorhergehende Entscheidungen in dieser Richtung ${ }^{81}$. Es handelte sich um Allgemeine Geschäftsbedingungen über Verteilung zwischen RS Components Limited und RS do Brasil Comércio, Importação, Exportação, Consultoria e Representações Ltda., in dem ausdrücklich der Gerichtsstand des Vereinigten Königreichs als kompetent zur Klärung eventueller Streitigkeiten gewählt wurde. Das Gericht verstand die Nichtfortführung des Streits zumal die Verteilungsverpflichtung - obwohl im Ausland festgelegt - in Brasilien

79 Oberster Gerichtshof, Revision Nr. 505208 AM 2003/0042379-0. Berichterstatter Minister Carlos Alberto Menezes Direito. Dritter Senat. DJ vom 13.10.2003, Seite 363, RDR Band 29, Seite 343, RSTJ Band 187, Seite 304.

80 Oberster Gerichtshof, Revision Nr. 242.383/SP, Berichterstatter Minister Humberto Gomes de Barros, dritter Senat. DJ vom 21.03.2005, Seite 360.

81 Oberster Gerichtshof, Revision Nr. 804306/SP, Berichterstatterin Ministerin Nancy Andrighi, drittes Senat, DJe vom 03.09.2008. 
zu erfüllen war, was der Annahme des Artikels 88, Absatz II entspricht und unveränderlich ist ${ }^{82}$.

Bezugnehmend auf einen internationalen Importvertrag garantierte nochmals der Oberste Gerichtshof (STJ) im Jahre 2010 die Anwendung der Gerichtsstandwahlklausel und meinte, auf der Grundlage vorhergehender Entscheidungen, dass die Wahl des Gerichtsstandes gültig ist, vorausgesetzt, dass sie das öffentliche Interesse nicht verletzt. Im vorliegendem Falle wurde Bologna (Italien) - Ort an dem der Vertrag geschlossen wurde - als gültiger Gerichtsstand und Durchführungsort der Verpflichtung anerkannt, da die Prozessfragilität der Rechtsmittelklägerin nicht identifiziert und auch kein Hindernis zum Justizzugang der Partei festgestellt wurde.

Schließlich erschien die neulich getroffene Entscheidung des Obersten Gerichtshofes (STJ) über die Nichtanerkennung der Gerichtsstandwahlklausel, die Spanien als zuständig erklärte ${ }^{84}$. Es handelt sich um die Möglichkeit einer natürlichen, in Brasilien wohnhaften Person, die brasilianische Gerichtsbarkeit anzurufen in einer Angelegenheit der vertraglichen Dienstleistung mit der Gerichtsstandklausel in jenem Lande. Die Klägerin stellte fest, dass ihre Erscheinung in unangebrachter Weise über eine ausländische elektronische Internetseite weltweit ausgestrahlt wurde und reichte eine Klage in Brasilien ein mit der Forderung immaterieller und materieller Entschädigung. Das Oberste Justizgericht erkennt die konkurrierende Zuständigkeit der brasilianischen Gerichtshoheit auf der Grundlage des Artikels 88, Absatz III der Zivilprozessordnung, zumal die schädigende Tat gegenüber der Erscheinung der in Brasilien wohnhaften Klägerin auch in Brasilien geschah unter der Voraussetzung, dass die Bilder der Autorin nicht nur in Spanien sondern weltweit abgerufen werden konnten. Das Oberste Justizgericht fügte hinzu, dass die Gerichtsstandwahlklausel des im Ausland abgeschlossenen Dienstleistungsvertrages - trotz der Annahme durch die brasilianische

82 Viele Autoren sind der Meinung, dass die Klausel der Gerichtstand-Wahl in diesem Falle nicht vorrangig ist, nicht wegen den vom Gericht genannten Gründen, sondern weil es sich um allgemeinen Geschäftsbedingungen handelte. Wenn die Gerichtstandwahl-Klausel von Natur aus vereinbarend ist, so könnte niemals eine ausdrücklich zwischen den Parteien vereinbarte Klausel Bestandteil allgemeiner Geschäftsbedingungen sein der, wiederum, die Natur eines bereits vereinbarten Vertrages hat, der von dem Lieferant der Dienstleistung oder des Produktes vorgefasst wurde und deshalb auch keinen vorherigen Verhandlungen oder nachträglichen Änderungen seiner bereits festgelegten Klauseln unterliegt. Andere Autoren sehen wiederum kein Hindernis in dieser Klausel im Falle der allgemeinen Geschäftsbedingungen, mit Ausnahme jedoch der Verbraucherverträge.

83 Oberster Gerichtshof, Revision Nr. 1177915/RJ, Berichterstatter Minister Vasco Della Giustina, dritter Senat. DJe vom 24.08.2010.

84 Oberster Gerichtshof, Revision Nr. 1168547/RJ, Berichterstatter Minister Luis Felipe Salomão, vierter Senat, DJe vom 07.02.2011. 
Judikative - kein Hindernis zur Klageeinreichung in Brasilien darstellt, trotz der konkurrenzfähigen Zuständigkeit. Schließlich verstärkte das Justizgericht seine konträre Einstellung gegenüber der Klausel und bekräftigte, dass die Entschädigungsklage der Klägerin nicht auf den Vertrag beruht, sondern auf Bildern und Erscheinungen die von der Angeklagten - ohne Zustimmung der Klägerin - benutzt wurden. Daher hat die Klausel gemäß Artikel 88 der Zivilprozessordnung keine Geltung.

Nach all diesem Vortrag ist die Feststellung möglich, dass der Oberste Gerichtshof (STJ) seine Meinung über die Annahme der ausländischen Gerichtsstandwahlklausel offen hält. Die opinio juris des Justizgerichts hat den Anschein nicht stabil zu sein. Dies bewirkt momentane Zweifel bei den Parteien bezüglich der ausländischen Vertragsabschlüsse.

In Bezug auf das Thema präsentiert das Projekt einer neuen Zivilprozessordnung jedoch einige technische Fortschritte. Die Vorschrift über die internationale Zuständigkeit, wie angedeutet, lässt die Hypothese der ausdrücklichen oder stillschweigenden Unterordnung unter die brasilianische Gerichtsbarkeit zu und eröffnet so zweifelsfrei die Möglichkeit der Bestimmung der Gerichtsbarkeit.

Außerdem führte der Gesetzgeber eine neue Vorschrift hinzu, mit der die brasilianische Gerichtsbarkeit von der Bearbeitung und die Entscheidungsfällung der Prozesse befreit wird, wenn in einem Vertrag eine ausländische Gerichtsstandwahlklausel steht, die von dem Beklagten in seiner Klageerwiderung in Frage gestellt wird ${ }^{85}$. Im Gegensatz zur jetzigen Zivilprozessordnung, wird auf diese Weise eine ausdrückliche Behandlung der Gerichtsstandwahl im Falle der ändernden Gerichtsbarkeit gegeben. Der Einzelparagraph der neuen Verordnung stellt jedoch klar, dass eine Änderung der brasilianischen Gerichtsbarkeit in den Annahmen der ausschließlichen ausländischen Zuständigkeiten nicht möglich ist.

Das Kapitel über interne Zuständigkeit enthält Anordnungen über die Wahl des brasilianischen Gerichtsstandes gemäß der aktuellen Vorschrift des Artikels 111 der Zivilprozessordnung ${ }^{86}$. Es ist angebracht zu erwähnen, dass während der Diskussionen über das Projekt erwägt wurde, die Gerichtsstandwahl in Allgemeinen Geschäftsbedingungen

85 Es gehört nicht zur brasilianischen Kompetenz die Prozessbearbeitung und -entscheidung, wenn in einem Vertrag eine ausländische Gerichtsstandwahlklausel steht die von dem Beklagten in seiner Klageerwiderung in Frage gestellt wird. Einzelparagraph - Die im „Caput“ dieses Artikels vorgeschriebene Anordnung findet keine Anwendung in Sachen der internationalen Kompetenz dieses Kapitels.

86 Die Parteien können die Kompetenz aufgrund des Wertes und des Ortes ändern, in dem sie den Gerichtsstand wählen an dem die Klagen bezüglich Rechte und Verpflichtungen eingereicht werden. 
zu verbieten; dieser Vorschlag wurde abgelehnt. Als Alternative schließt der Gesetzgeber eine generelle Annahme ein bezüglich einer generellen Erklärung über die Unwirksamkeit der Gerichtsstandwahl in allen Fällen, in denen sie sich missbräuchlich zeigt ${ }^{87}$. Es handelt sich jedoch um eine Anordnung des Kapitels der internen Zuständigkeit. Ihre Anwendung bei der Wahl eines ausländischen Gerichtsstandes bleibt ungeklärt.

In gleicher Weise äußerte sich der Gesetzgeber nicht ausdrücklich über die eventuelle Wahl eines ausländischen Gerichtsstandes in Allgemeinen Geschäftsbedingungen über Verbraucherbeziehungen. Es hat den Anschein, dass diese Annahme möglich ist unter Berücksichtigung, dass dieEntscheidungszuständigkeitin Prozessenüber die Verbraucherbeziehungen nicht ausdrücklich mit der brasilianischen Exklusivität fixiert wurde. Dies könnte ab einer systematischen Auslegung dazu führen, dass die brasilianische Gerichtsbarkeit durch eine ausländische austauschbar wäre. Es sei nochmals ausdrücklich erwähnt, dass die Ablehnung einer missbräuchlichen Gerichtsstandwahl nur im Bereich der internen Zuständigkeit vorgesehen ist.

\section{SCHLUSSBETRACHTUNG}

Das Projekt der Zivilprozessordnung hat zum Ziel, einige Unstimmigkeiten und Kontroversen des aktuellen Prozessmodus der internationalen Zuständigkeit und Gerichtsstandwahl auszubessern. Durch die neue Redaktion erklärt der Gesetzgeber, dass die Annahmen der konkurrierenden internationalen Zuständigkeiten nicht kumulativ sind. Der Gesetzgeber scheint auch die technisch unkorrekte Anwendung des Ausdrucks ,,internationale Zuständigkeit“ sanieren zu wollen.

In Bezug auf die vorhandenen Vorgaben der jetzigen Zivilprozessordnung, präsentiert das Projekt ein Minimum an Redaktionsänderungen. Ausnahme hierzu bildet die Hinzufügung einer neuen Vorschrift mit drei Hypothesen zur Ausübung der brasilianischen Gerichtsbarkeit, wobei jedoch nicht geklärt wird, ob diese Hypothesen ausschließlich oder konkurrierend sind. Während unserer Studie konnten wir einige praktische Konsequenzen bei der Erwägung einer jeder Annahme der neuen Vorschrift betrachten, ungeachtet ihres konkurrierenden oder ausschließlichen Charakter.

Das Projekt stellt mit Sicherheit einen großen Fortschritt dar, indem es ausdrücklich erlaubt, dass die Parteien den brasilianischen oder jedweden ausländischen Gerichtsstand zur Beseitigung von Interessenkonflikte in den Vertragsbeziehungen auswählen dürfen.

87 Die Gerichtsstandwahlklausel kann von Amts wegen vor der Ladung ungültig erklärt werden wenn sie missbräuchlicher Natur ist. Zu diesem Zeitpunkt befiehlt der Richter die Weiterleitung des Prozesses an das Gericht des Wohnsitzes des Angeklagten. 
Diese Möglichkeit schließt die derzeitigen Unsicherheiten der Rechtsprechung aus und erlaubt somit, dass Brasilien eine hohe Position als Konkurrent im Außenhandel erreichen kann.

Es ist aber nicht zu übersehen, dass tatsächlich das Projekt einige der alten interpretativen Kontroversen aufzeigt, indem es die aktuelle Redaktion der Zivilprozessordnung beibehält. In den Verbraucherbeziehungen erweckt es in der neuen Form neue Fragestellungen: nicht nur in Bezug auf internationale Zuständigkeiten, sondern auch und tatsächlich in Bezug auf die Wahlmöglichkeit eines ausschließlichen ausländischen Gerichtsstandes. Ein weiterer Punkt ist die Redaktionsausdehnung, die erlaubt, dass die Parteien sich formell oder stillschweigend der brasilianischen Gerichtsbarkeit unterwerfen können, ohne, dass es ein Verbindungselement zwischen dem Rechtsstreit und dem Gerichtsstand bedarf.

Selbst wenn die durch das Projekt der Zivilprozessordnung einzuführenden Änderungen Fortschritte des internationalen Zivilverfahrensrechts für Brasilien darstellen können, so werden doch verschiedene Fragen über die Anwendbarkeit der neu anzuwendenden Vorgaben des Gesetzgebers forterhalten bleiben.

\section{LITERATURVERZEICHNIS}

ARAUJO, Nadia de. Direito Internacional Privado: Teoria e Prática Brasileira. Rio de Janeiro: Renovar, 2008.

BARBI, Celso Agricola. Comentários do Código de Processo Civil. 9. Auflage. Rio de Janeiro: Forense, 1994.

CINTRA, Antonio Carlos de Araújo; GRINOVER, Ada Pellegrini; DINAMARCO, Cândido Rangel. Teoria Geral do Processo. 23. Auflage. São Paulo: Malheiros, 2007.

GRECO, Leonardo. A competência internacional da justiça brasileira. [Die internationale Kompetenz der brasilianischen Justiz]. Revista da

Faculdade de Direito de Campos, Jahr VI, Nr. 7, Dezember 2005. MADRUGA, Antenor. Homologação de sentença estrangeira de falência. Verfügbar in: http:/www.conjur.com.br/2011-jun-22/ judiciario-estrangeiro-nao-decretar-falencia-empresa-brasileira. Abgerufen am 23.01.2013.

MELLO, Celso D. de Albuquerque. Direito Internacional Econômico. Rio de Janeiro: Renovar, 2003.

MESQUITA, José Ignácio Botelho de. Da competência internacional e dos princípios que a informam [Über die internationale Kompetenz und ihre Informationsprinzipien]. Revista de Processo, Nr. 50, 1988.

MOREIRA, José Carlos Barbosa. Probleme in Bezug auf internationale Streitigkeiten. In Temas de Direito Processual. 5. Serie. Rio de Janeiro: Saraiva, 1994. 
PONTES DE MIRANDA, Francisco Cavalcanti. Comentários ao Código de Processo Civil, Band II, Rio de Janeiro: Forense, 1973.

ROBERTO, Wilson Furtado. Dano Transnacional e internet: direito aplicável e competência internacional. Curitiba: Juruá, 2010.

STRENGER, Irineu. Contratos Internacionais do Comércio, 2. Auflage, São Paulo: Editora Revista dos Tribunais, 1992.

TIBURCIO, Carmen. Temas de Direito Internacional. Rio de Janeiro: Renovar, 2006.

TORNAGHI, Hélio. Comentários ao Código de Processo Civil. Band 1, São Paulo: RT, 1974.

VALLADÃO, Haroldo, Direito Internacional Privado, Band III, Rio de Janeiro: Freitas Bastos, 1978. 